\title{
Fuzzy Left Almost Semihyperring
}

\author{
S I Rahman ${ }^{1, *}$, N Hidayat $^{2}$, and A R Alghofari ${ }^{2}$ \\ ${ }^{1}$ Student of Master Mathematics Study Program, University of Brawijaya, Indonesia \\ ${ }^{2}$ Department of Mathematics, Faculty of Mathematics and Science, University of Brawijaya, Indonesia \\ *Corresponding author. Email: shintarahman15@student.ub.ac.id
}

\begin{abstract}
The left almost semihyperring (LA) is an algebraic hyperstructure satisfying two axioms, these are the left inverse hyperoperation and the distributive axiom between multiplication and addition hyperoperation. In this article, the concept of fuzzy sets is applied to these structures so that we get a new algebraic structure, and it is called fuzzy left almost semihyperring. We show that the set of all fuzzy subsets in LA-semihyperring is also LA-semihyperring. Furthermore, the LA-subsemihyperring and hyperideal fuzzy properties and their relationship to the characteristic function and level set are analyzed.
\end{abstract}

Keywords: LA-semihyperrings, Subset Fuzzy, Fuzzy Hyperideals.

\section{INTRODUCTION}

Mathematics as a universal language for the analysis, simulation, optimization and control of an industrial process. Mathematical abstraction is able to provide solutions to practical problems that stimulate new findings. The development of technology and communication in this era grows rapidly and there are many interconnections between algebra and science technology and communication, such as cryptography, algorithmic efficiency analysis, geometric structures, automata theory, and also in the concept of probability. Some of these algebraic fields are concepts of algebraic hyperstructure that introduced by the French mathematician, Marty[1] and fuzzy set theory which were first introduced by Zadeh[2]. Nowdays, Fuzzy set theory has various applications in areas such as computer engineering, artificial intelligence, control engineering, operations research, management science, robots and many more.

A number of articles have applied fuzzy concepts to algebraic structures and algebraic hyperstructures. Rosenfeld[3] applied the fuzzy concept to groups. Kuroki[4] introduced the idea of fuzy ideal and fuzzy biideal to semigroups. Leoreanu and Davvaz[5] introduced fuzzy hyperring. Ahsan et al.[6] discussed fuzzy semiring and its applications. The fuzzy semihypergroup introduced by Sen et al.[7]. Dutta et al.[8] introduced the idea of fuzzy prime and ideal prime in semihyperring.
Kehayopulu[9] discussed fuzzy semihypergroups. The purpose of this article is to apply the fuzzy concept to a hyperstructure algebra called "left almost (LA) semihyperring" that introduced by Nawaz et al.[10] We define the notion of fuzzy LA-semihyperring and analyze its relationship to LA-semihyperring and discuss its hyperideal properties and its relation to t-levels and characteristic functions.

\section{PRELIMINARIES}

This research uses literature study in several scientific books and journals related to algebraic hyperstructures and the concept of fuzzy sets. We analyze the definitions and properties of the previous structure to develop the LA-semihyperring fuzzy structure. Some basic terminology from the literature on left Almost (LA) semihyperring and fuzzy sets is presented in this section.

Definition 2.1.[10] Let $H$ be the non-empty set and $\mathcal{P}^{*}(H)$ the set of all non-empty subsets of $H$. Binary hyperoperation " $\bigoplus$ " is a mapping defined as follows.

$$
\begin{gathered}
\bigoplus: H \times H \rightarrow \mathcal{P}^{*}(H) \\
(a, b) \rightarrow \bigoplus(a, b)=a \oplus b
\end{gathered}
$$

The structure $(H, \oplus)$ is called hypergroupoid.

Definition 2.2.[10] Let $A, B \subseteq H$ are non-empty set and $x \in H$. The hyperoperation " $\bigoplus$ " on $A, B,\{x\} \in \mathcal{P}^{*}(H)$ is defined as follows.

(i) $A \oplus B=\bigcup_{a \in A, b \in B}(a \oplus b)$ 
(ii) $A \oplus x=A \oplus\{x\}$.

(iii) $x \oplus B=\{x\} \oplus B$

Definition 2.3.[10] A hypergroupoid $(H, \oplus)$ is called LA-semihyprgroup if for all $x, y, z \in G$ satisfies the left inverted axiom as follows.

Which means that

$$
(x \oplus y) \oplus z=(z \oplus y) \oplus x
$$

$$
\bigcup_{u \in(x \oplus y)} u \oplus z=\bigcup_{v \in(z \oplus y)} v \oplus x
$$

Definition 2.4.[10] An algebraic hyperstructure $(R, \circ, *)$ is said to be a LA-semihyperring if it is satisfies the following axioms.

(i) $(R, \circ)$ is a LA-semihypergroup.

(ii) $(R, *)$ is a LA-semihypergroup with the absorbing element that is "0" $(0 * x=x * 0=0)$ for every $x \in$ $R$.

(iii) The hyperoperation " * " is distributive with respect to the hyperoperation " $\circ$ " for all $x, y, z \in R$, that is

(iv) $x *(y \circ z)=(x * y) \circ(x * z)$ and $(y \circ z) * x=$ $(y * x) \circ(z * x)$.

Definition 2.5.[10] Let $R$ be an LA-hypersemiring. An element $e \in R$ is called

(i) Left identity (resp., pure left identity) if for all $x \in$ $R, x \in e * x$ (resp., $x=e \circ x$ )

(ii) Right identity (resp., pure right identity) if for all $x \in$ $R, x \in x * e$ (resp., $x=x \circ e$ )

(iii) Identity (resp., pure identity) if for all $x \in R, x \in e *$ $x \cap x * e$ (resp., $x=e \circ x \cap x * e)$

\section{FUZZY LA SEMIHYPERRING}

This section present the definition and some basic properties of fuzzy LA-semihyperring and its hyperideal

Definition 3.1. Let $(R, \oplus, \odot)$ be an LA-semihyperring. A fuzzy subset $f$ of $R$ is a function that defined as follow.

$$
f: R \mapsto[0,1]
$$

Definition 3.2. Let $F(R)$ be a set of all fuzzy subset on LA-semihyperring $(R, \oplus, \odot)$. Hyperoperation " + " and "。" on $F(R)$ are defined as follows.

$$
(f+g)(x)=\left\{\begin{aligned}
\bigvee_{x \in(y \oplus z)}\{f(y) \wedge g(z)\}, & x \in y \oplus z \\
0, & x \notin y \oplus z
\end{aligned}\right.
$$

and

$$
(f \circ g)(x)=\left\{\begin{aligned}
\bigvee_{x \in\left(\sum_{i=1}^{n} y_{i} \odot z_{i}\right)}\left\{\bigwedge_{1}^{n} f\left(y_{i}\right) \wedge g\left(z_{i}\right)\right\}, & x \in y \oplus z \\
0, & x \notin y \oplus z
\end{aligned}\right.
$$

for every $f, g \in F(R)$ and $x, y, z \in R$.

Theorem 3.3. If $(R, \oplus, \odot)$ is an LA-semihyperring then $(F(R),+, \circ)$ is an LA-semihyperring.

Proof. Let $f, g, h \in F(R)$ and $x \in R$ such that $x \notin y \circ z$ for some $y \circ z$ then

$$
[(f+g)+h](x)=0=[(h+g)+f](x) .
$$

Let $x \in y \oplus z$ then

$$
\begin{aligned}
{[(f+g)+h](x) } & \left.=\bigvee_{x \in y \oplus z}\{f+g)(y) \wedge h(z)\right\} \\
& =\bigvee_{x \in y \oplus z}\left\{\bigvee_{y \in a \oplus b}\{f(a) \wedge g(b)\} \wedge h(z)\right\} \\
& =\bigvee_{x \in(a \oplus b) \oplus z}\{f(a) \wedge g(b) \wedge h(z)\} \\
& =\bigvee_{x \in(a \oplus b) \oplus z}\{f(a) \wedge g(b) \wedge h(z)\} \\
& =\bigvee_{x \in(z \oplus b) \oplus a}\{h(z) \wedge g(b) \wedge f(a)\} \\
& =\bigvee_{x \in(z \oplus b) \oplus a}\{h(z) \wedge g(b) \wedge f(a)\} \\
& =\bigvee_{x \in p \oplus a}\left\{\bigvee_{p \in(z \oplus a)}\{h(z) \wedge g(b)\} \wedge f(a)\right\} \\
& =\bigvee_{x \in p \oplus a}\{(h+g)(p) \wedge f(a)\} \\
& =[(h+g)+f](x) .
\end{aligned}
$$

Therefore, $(F(R),+)$ is an LA-hypersemigroup. Let $x \notin$ $\sum_{i=1}^{n} y_{i} \odot z_{i}$ then

$$
[(f \circ g) \circ h](x)=0=[(h \circ g) \circ f](x) \text {. }
$$

Let $x \in \sum_{i=1}^{n} y_{i} \odot z_{i}$ then

$$
\begin{aligned}
& {[(f \circ g) \circ h](x)=\bigvee_{x \in \sum_{i=1}^{n} y_{i} \odot z_{i}}\left\{\bigwedge_{1}^{n}(f \odot g)\left(y_{i}\right) \wedge h\left(z_{i}\right)\right\}} \\
& =\bigvee_{x \in \sum_{i=1}^{n} y_{i} \odot z_{i}}\left\{\bigwedge_{1}^{n}\left\{\bigvee_{y_{i} \in \sum_{i=1}^{n} a_{i} \odot b_{i}}\left\{\bigwedge_{1}^{n} f\left(a_{i}\right) \wedge g\left(b_{i}\right)\right\} \wedge h\left(z_{i}\right)\right\}\right\} \\
& =\bigvee_{x \in \sum_{i=1}^{n}\left(\sum_{i=1}^{n} a_{i} \odot b_{i}\right) \odot z_{i}}\left\{\bigwedge_{1}^{n}\left\{\bigwedge_{1}^{n} f\left(a_{i}\right) \wedge g\left(b_{i}\right)\right\} \wedge h\left(z_{i}\right)\right\} \\
& =\bigvee_{x \in \sum_{i=1}^{n}\left(\sum_{i=1}^{n} a_{i} \odot b_{i}\right) \odot z_{i}}\left\{\bigwedge_{1}^{n}\left\{\bigwedge_{1}^{n} f\left(a_{i}\right) \wedge g\left(b_{i}\right)\right\} \wedge h\left(z_{i}\right)\right\} \\
& =\bigvee_{x \in \sum_{i=1}^{n}\left(a_{i} \odot b_{i}\right) \odot z_{i}}\left\{\bigwedge_{1}^{n}\left(f\left(a_{i}\right) \wedge g\left(b_{i}\right)\right) \wedge h\left(z_{i}\right)\right\} \\
& =\bigvee_{x \in \sum_{i=1}^{n}\left(a_{i} \odot b_{i}\right) \odot z_{i}}\left\{\bigwedge_{1}^{n}\left(f\left(a_{i}\right) \wedge g\left(b_{i}\right)\right) \wedge h\left(z_{i}\right)\right\} \\
& =\bigvee_{x \in \sum_{i=1}^{n}\left(z_{i} \odot b_{i}\right) \odot b_{i}}\left\{\bigwedge_{1}^{n}\left(h\left(z_{i}\right) \wedge g\left(b_{i}\right)\right) \wedge f\left(a_{i}\right)\right\} \\
& =\bigvee_{x \in \sum_{i=1}^{n}\left(z_{i} \odot b_{i}\right) \odot b_{i}}\left\{\bigwedge_{1}^{n}\left(h\left(z_{i}\right) \wedge g\left(b_{i}\right)\right) \wedge f\left(a_{i}\right)\right\} \\
& =\bigvee_{x \in \sum_{i=1}^{n}\left(\sum_{i=1}^{n} z_{i} \odot b_{i}\right) \odot a_{i}}\left\{\bigwedge_{1}^{n}\left\{\bigwedge_{1}^{n} h\left(z_{i}\right) \wedge g\left(b_{i}\right)\right\} \wedge f\left(a_{i}\right)\right\} \\
& =\bigvee_{x \in \sum_{i=1}^{n} p_{i} \odot a_{i}}\left\{\bigwedge_{1}^{n}\left\{\bigvee_{y_{i} \in \sum_{i=1}^{n} \odot \odot a_{i}}\left\{\bigwedge_{1}^{n} h\left(z_{i}\right) \wedge g\left(b_{i}\right)\right\} \wedge f\left(a_{i}\right)\right\}\right\} \\
& =\bigvee_{x \in \sum_{i=1}^{n} p_{i} \odot a_{i}}\left\{\bigwedge_{1}^{n}(h \odot g)\left(p_{i}\right) \wedge f\left(a_{i}\right)\right\}=[(h \circ g) \circ f](x) \text {. }
\end{aligned}
$$

Now, we will prove that " + "distributive to "。".

$$
\begin{aligned}
& {[f \circ(g+h)](x)=\bigvee_{x \in \sum_{i=1}^{n} y_{i} \odot z_{i}}\left\{\bigwedge_{1}^{n}\left(f\left(y_{i}\right) \wedge(g+h)\left(z_{i}\right)\right)\right\}} \\
& =\bigvee_{x \in \sum_{i=1}^{n} y_{i} \odot z_{i}}\left\{\bigwedge_{1}^{n}\left\{f\left(y_{i}\right) \wedge \bigvee_{z_{i} \in a \oplus b}\{g(a) \wedge h(b)\}\right\}\right\}
\end{aligned}
$$




$$
\begin{aligned}
& =\bigvee_{x \in \sum_{i=1}^{n} y_{i} \odot(a \oplus b)}\left\{\bigwedge_{1}^{n} f\left(y_{i}\right) \wedge(g(a) \wedge h(b)\}\right. \\
& =\bigvee_{x \in\left(\sum_{i=1}^{n} y_{i} \odot a\right) \oplus\left(\sum_{i=1}^{n} y_{i} \odot b\right)}\left\{\left\{\bigwedge_{1}^{n}\left(f\left(y_{i}\right) \wedge g(a)\right)\right\} \wedge\left\{\bigwedge_{1}^{n}\left(f\left(y_{i}\right) \wedge h(b)\right)\right\}\right\} \\
& =\bigvee_{x \in p \oplus q}\left\{\bigvee_{p \in \sum_{i=1}^{n} y_{i} \odot a}\left\{\bigwedge_{1}^{n}\left(f\left(y_{i}\right) \wedge g(a)\right)\right\} \wedge \bigvee_{q \in \sum_{i=1}^{n} y_{i} \odot b}\left\{\bigwedge_{1}^{n}\left(f\left(y_{i}\right) \wedge h(b)\right)\right\}\right\} \\
& =\bigvee_{x \in p \oplus q}\{(f \circ g)(p) \wedge(f \circ h)(q)\}=[(f \circ g)+(f \circ h)](x) .
\end{aligned}
$$

With analog way to prove right distributive. $F(R)$ is left inverted and distributive such that $(F(R),+, \circ)$ is an LA-semihyperring. $\square$

Theorem 3.4. If $(F(R),+, \circ)$ is an LA-semihyperring then the medial law is hold in $F(R)$.

Proof. Straightforward

Theorem 3.5. If $(R, \oplus, \odot)$ is an LA-semihyperring with a pure left identity then the following axioms are hold.

(i) $(f \circ g) \circ(h \circ k)=(k \circ h) \circ(g \circ f)$

(ii) $f \circ(g \circ h)=g \circ(f \circ h)$.

For any $f, g, h, k \in F(R)$.

Proof. Striaghtforward.

Theorem 3.6. An LA-semihyperring $F(R)$ is a semihyperring if only if $f \circ(g \circ h)=(h \circ g) \circ f$ for all $f, g, h, \in F(R)$.

Proof. Straightforward.

Theorem 3.7. An LA-semihyperring $F(R)$ is a commutative semihyperring if only if $(f \circ g) \circ h=f \circ$ $(h \circ g)$.

Proof. Straightforward.

Definition 3.8. Let $(R, \oplus, \odot)$ be an LA-semihyperring. A fuzzy subset $f$ is a fuzzy LA-subsemihyperring of $R$ if the following axioms are hold.

(i) $\bigwedge_{z \in x \oplus y} f(z) \geq \min \{f(x), f(y)\}$

(ii) $\wedge_{z \in x \odot y} f(z) \geq \min \{f(x), f(y)\}$.

For all $x, y, z \in R$.

Definition 3.9. Let $(R, \oplus, \odot)$ be an LA-semihyperring. A fuzzy subset $f$ is hyperideal of $R$ if the following axioms are hold.
(i) $\wedge_{z \in x \oplus y} f(z) \geq \min \{f(x), f(y)\}$
(ii) $\wedge_{z \in x \odot y} f(z) \geq\{f(y)\}$ (left hyperideal)
(iii) $\bigwedge_{z \in x \odot y} f(z) \geq\{f(x)\}$ (right hyperideal)
(iv) $\bigwedge_{z \in x \odot y} f(z) \geq \max \{f(x), f(y)\}$ (hyperideal).
For all $x, y, z \in R$

An LA-semihyperring $R$ can be considered a fuzzy subset of itself notated as $\boldsymbol{R}$ and defined as follows.

$$
\begin{aligned}
\boldsymbol{R} & : R \mapsto[0,1] \\
& : x \mapsto \boldsymbol{R}(x)=1
\end{aligned}
$$

Lemma 3.10. If $(R, \oplus, \odot)$ be an LA-semihyperring with a pure left identity then $\boldsymbol{R} \circ \boldsymbol{R}=\boldsymbol{R}$.

Proof. Let $x \in R$ and $e$ be a pure left identity in $R$ such that $x \in e \odot x$. Thus,

$$
\begin{aligned}
(\boldsymbol{R} \circ \boldsymbol{R})(x)= & \bigvee_{\substack{x \in \sum_{i=1}^{n} a_{i} \odot b_{i} \\
\geq \boldsymbol{R}(e) \wedge}}\left\{\bigwedge_{1}^{n}\{\boldsymbol{R}(x)=1=\boldsymbol{R}(x) .\right.
\end{aligned}
$$

Theorem 3.11. Let $f$ be a fuzzy subset of an LAsemihyperring $R$ the the following statements are true.

(i) A fuzzy subset $f$ is a fuzzy LA-subsemihyperring $R$ if only if $f+f \subseteq f$ and $f \circ f \subseteq f$.

(ii) A fuzzy subset $f$ is a fuzzy left hyperideal of LAsemihyperring $R$ if only if $f+f \subseteq f$ and $\boldsymbol{R} \circ f \subseteq f$.

(iii) A fuzzy subset $f$ is a fuzzy right hyperideal of LAsemihyperring $R$ if only if $f+f \subseteq f$ and $f \circ \boldsymbol{R} \subseteq f$.

Proof. Let $f$ and $\boldsymbol{R}$ be subset fuzzy of an LAsemihyperring $R$.

(i) let $f$ be a fuzzy LA-subsemihyperring $R$.

$$
(f+f)(z)=\bigvee_{x \in a \oplus b}\{f(a) \wedge f(b)\} \leq \bigvee_{x \in a \oplus b} f(a \oplus b)=f(z)
$$

and

$$
\begin{aligned}
(f \circ f)(z)=\bigvee_{x \in \sum_{i=1}^{n} a_{i} \odot b_{i}}\left\{\bigwedge_{1}^{n} f\left(a_{i}\right) \wedge f\left(b_{i}\right)\right\} \\
\left.\leq \bigvee_{x \in \sum_{i=1}^{n} a_{i} \odot b_{i}}\left\{\bigwedge_{1}^{n} f\left(a_{i} \odot b_{i}\right)\right)\right\}=f(z)
\end{aligned}
$$

Conversely, Let $f+f \subseteq f$ and $f \circ f \subseteq f$.

$\bigwedge_{z \in x \oplus y} f(z) \geq \bigwedge_{z \in x \oplus y}(f+f)(z)=(f+f)(x \oplus y)$

$$
=\bigvee_{z \in(x \oplus y) \in(a \oplus b)} f(a) \wedge f(b) \geq f(x) \wedge f(y)
$$

It means

$$
\bigwedge_{z \in x \oplus y} f(z) \geq \min \{f(x), f(y)\}
$$

Next,

$$
\begin{aligned}
\bigwedge_{z \in x \odot y} f(z) \geq \bigwedge_{z \in x \odot y}(f \circ f)(z)=(f \circ f)(x \odot y) \\
=\bigvee_{z \in(x \odot y) \in \sum_{i=0}^{n} a_{i} \odot b_{i}}\left\{\bigwedge_{1}^{n}\left(f\left(a_{i}\right) \wedge f\left(b_{i}\right)\right\}\right. \\
\geq f(x) \wedge f(y)
\end{aligned}
$$

It means

$$
\bigwedge_{z \in x \odot y} f(z) \geq \min \{f(x), f(y)\}
$$

(ii) Let $f$ be a fuzzy left hyperideal of LA-semihyperring $R$ and $x \in R$.

$$
\begin{aligned}
& (\boldsymbol{R} \circ f)(\mathbf{z})=\bigvee_{z \in \sum_{i=1}^{n} a_{i} \odot b_{i}}\left\{\bigwedge_{1}^{n}\left\{\boldsymbol{R}\left(a_{i}\right) \wedge f\left(b_{i}\right)\right\}\right\} \\
& =\bigvee_{z \in \sum_{i=1}^{n} a_{i} \odot b_{i}}\left\{\bigwedge_{1}^{n}\left\{1 \wedge f\left(b_{i}\right)\right\}\right\}=\bigvee_{z \in \sum_{i=1}^{n} a_{i} \odot b_{i}}\left\{\bigwedge_{1}^{n}\left\{f\left(b_{i}\right)\right\}\right\} \\
& \leq \bigvee_{z \in \sum_{i=1}^{n} a_{i} \odot b_{i}}\left\{\bigwedge_{1}^{n}\left\{f\left(a_{i} \odot b_{i}\right)\right\}\right\}=f(z)
\end{aligned}
$$

Conversely, let $\boldsymbol{R} \circ f \subseteq f$ then

$$
\begin{aligned}
\bigwedge_{z \in x \odot y} f(z) \geq \bigwedge_{z \in x \odot y}(\boldsymbol{R} \circ f)(z)=(\boldsymbol{R} \circ f)(x \odot y) \\
=\bigvee_{z \in(x \odot y) \in \sum_{i=0}^{n} a_{i} \odot b_{i}}\left\{\bigwedge_{1}^{n}\left(\boldsymbol{R}\left(a_{i}\right) \wedge f\left(b_{i}\right)\right\}\right. \\
\geq \boldsymbol{R}(x) \wedge f(y)=1 \wedge f(y) \geq f(y) .
\end{aligned}
$$


It means that $f$ a fuzzy hyperideal of $R$. With analog way to prove (iii).

Theorem 3.12. Let $(R, \oplus, \odot)$ be an LA-semihyperring with pure left identity. If $f, g \in F(R)$ are fuzzy LAsubsemihyperring of $R$ then $f \circ g$ is fuzzy LAsubsemihyperring of $R$.

Proof. Firstly, We will prove that $(f \circ g)(x) \wedge(f \circ$ $g)(y) \leq \Lambda_{z \in(x \circ y)}(f \circ g)(z)$.

$$
\begin{aligned}
& (f \circ g)(x) \wedge(f \circ g)(y) \\
& =\left[\bigvee_{x \in \sum_{i=1}^{n} a_{i} \odot b_{i}}\left\{\bigwedge_{1}^{n} f\left(a_{i}\right) \wedge g\left(b_{i}\right)\right\} \wedge\left[\bigvee_{x \in \Sigma_{i=1}^{n} c_{i} \odot d_{i}}\left\{\bigwedge_{1}^{n} f\left(c_{i}\right) \wedge g\left(d_{i}\right)\right\}\right]\right. \\
& =\bigvee_{x \in \sum_{i=1}^{n} a_{i} \odot b_{i}} \bigvee_{x \in \sum_{i=1}^{n} c_{i} \odot d_{i}}\left\{\bigwedge_{1}^{n} f\left(a_{i}\right) \wedge g\left(b_{i}\right) \wedge \bigwedge_{1}^{n} f\left(c_{i}\right) \wedge g\left(d_{i}\right)\right\} \\
& \leq \bigvee_{x \oplus y \in \sum_{i=1}^{n}\left(p_{i} \odot q_{i}\right)}\left\{\bigwedge_{1}^{n} f\left(p_{i}\right) \wedge g\left(q_{i}\right)\right\}=(f \circ g)(x \oplus y) \\
& =\bigwedge_{z \in x \oplus y}(f \circ g)(z)
\end{aligned}
$$

Secondly, since $F(R)$ is an LA-semihyperring then the medial law is satisfied so that

$$
(f \circ g) \circ(f \circ g)=(f \circ f) \circ(g \circ g) \subseteq f \circ g .
$$

Theorem 3.13. Let $(R, \oplus, \odot)$ be an LA-semihyperring with a pure identity. If $f, g \in F(R)$ are fuzzy hyperideal of $R$ then $f \circ g$ is a fuzzy hyperideal of $R$.

Proof. Based on Theorem 3.12, It is clear that $f \circ g$ satisfy the first axiom of Definition 3.9. Next, we will prove $f \circ g$ is a fuzzy hyperideal. Let $x \in \sum_{i=0}^{n} a_{i} \odot b_{i}$ and $e$ is a pure identity of $R$. By medial law we have

$$
\begin{gathered}
x \odot y=\sum_{i=1}^{n}\left(a_{i} \odot b_{i}\right) \odot y=\sum_{i=1}^{n}\left(a_{i} \odot b_{i}\right) \odot(e \odot y) \\
=\sum_{i=1}^{n}\left(a_{i} \odot e\right) \odot\left(b_{i} \odot y\right) .
\end{gathered}
$$

Since $f$ and $g$ are hyperideal of $R$ then

$$
\begin{aligned}
& (f \circ g)(x)=\bigvee_{x \in \sum_{i=1}^{n} a_{i} \odot b_{i}}\left\{\bigwedge_{1}^{n}\left\{f\left(a_{i}\right) \wedge g\left(b_{i}\right)\right\}\right\} \\
& \leq \bigvee_{x \odot y \in \sum_{i=1}^{n}\left(a_{i} \odot e\right) \odot\left(b_{i} \odot y\right)}\left\{\bigwedge_{1}^{n}\left\{f\left(a_{i} \odot e\right) \wedge g\left(b_{i} \odot y\right)\right\}\right\} \\
& \leq \bigvee_{x \odot y \in \sum_{i=1}^{n} c_{i} \odot d_{i}}\left\{\bigwedge_{1}^{n}\left\{f\left(c_{i}\right) \wedge g\left(d_{i}\right)\right\}\right\}=(f \circ g)(x \odot g) \\
& =\bigwedge_{z \in x \odot y}(f \circ g)(z)
\end{aligned}
$$

Thus, $f \circ g$ are a fuzzy right hyperideal of $R$. With analog way to prove fuzzy left hyperideal of $R$.

Theorem 3.14. Let $(R, \oplus, \odot)$ be an LA-semihyperring with pure identity. Every fuzzy right hyperideal of $R$ is a fuzzy hyperideal of $R$.

Proof. Let $e$ be a pure identity of $R$ and $f$ be a fuzzy right hyperideal of $R$. We will prove that $f$ is a fuzzy left hyperideal of $R$. By Lemma 3.10 we have

$$
\boldsymbol{R} \circ f=(\boldsymbol{R} \circ \boldsymbol{R}) \circ f=(f \circ \boldsymbol{R}) \circ \boldsymbol{R} \subseteq f \circ \boldsymbol{R} \subseteq \boldsymbol{f} .
$$

Theorem 3.15. Let $(R, \oplus, \odot)$ be an LA-semihyperring. If $\left\{f_{i}\right\}_{i \in \mathbb{N}}$ are fuzzy left hyperideal of $R$ then $\bigcap_{i \in \mathbb{N}} f_{i}$ is a fuzzy left hyperideal of $R$.

Proof. Let $f_{i}$ be fuzzy left hyperideal of $R$ for all $i \in \mathbb{N}$.

$$
\begin{aligned}
\bigcap_{i \in \mathbb{N}} f_{i}(x \oplus y)=\inf & \left\{f_{i}(x \oplus y)\right\} \geq \inf \left\{\min \left\{f_{i}(x), f_{i}(y)\right\}\right\} \\
= & \min \left\{\inf f_{i}(x), \inf f_{i}(y)\right\} \\
& =\min \left\{\left(\cap f_{i}\right)(x),\left(\cap f_{i}\right)(y)\right\}
\end{aligned}
$$

and

$\bigcap_{i \in \mathbb{N}} f_{i}(x \odot y)=\inf \left\{f_{i}(x \odot y)\right\} \geq \inf \left\{\min \left\{f_{i}(y)\right\}\right\}$

$$
=\min \left\{\inf f_{i}(y)\right\}=\min \left\{\left(\cap f_{i}\right)(y)\right\} .
$$

So, $\bigcap_{i \in \mathbb{N}} f_{i}$ is a fuzzy left hyperideal of $R$.

Theorem 3.16. Let $(R, \oplus, \odot)$ be an LA-semihyperring with pure left identity. If $f$ and $g$ are fuzzy hyperideal of $R$ then $f \circ g \subseteq f \cap g$.

Proof. Let $f, g \in F(R)$ are fuzzy hyperideal of $R$.

$$
\begin{aligned}
(f \circ g)(x)=\bigvee_{x \in \sum_{i=1}^{n} a_{i} \odot b_{i}}\left\{\bigwedge_{1}^{n}\left\{f\left(a_{i}\right) \wedge g\left(b_{i}\right)\right\}\right\} \\
\leq \bigvee_{x \in \sum_{i=1}^{n} a_{i} \odot b_{i}}\left\{\bigwedge_{1}^{n}\left\{f\left(a_{i} \odot b_{i}\right) \wedge g\left(a_{i} \odot b_{i}\right)\right\}\right\} \\
=\bigvee_{x \in \sum_{i=1}^{n} a_{i} \odot b_{i}}\left\{\bigwedge_{1}^{n}(f \cap g)\left(a_{i} \odot b_{i}\right)\right\} \\
=(f \cap g)(x) .
\end{aligned}
$$

Theorem 3.17. Let $(R, \oplus, \odot)$ be an LA-semihyperring. If $f$ is a fuzzy right hyperideal of $R$ then $f \circ f$ is a fuzzy hyperideal of $R$.

Proof. Let $f$ is a fuzzy right hyperideal of $R$.

$$
\begin{aligned}
& (f \circ f)(x)=\bigvee_{x \in \sum_{i=1}^{n} a_{i} \odot b_{i}}\left\{\bigwedge_{1}^{n}\left\{f\left(a_{i}\right) \wedge f\left(b_{i}\right)\right\}\right\} \\
& =\bigvee_{x \odot y \in \sum_{i=1}^{n}\left(a_{i} \odot b_{i}\right) \odot y}\left\{\bigwedge_{1}^{n}\left\{f\left(a_{i}\right) \wedge f\left(b_{i}\right)\right\}\right\} \\
& \leq \bigvee_{x \odot y \in \sum_{i=1}^{n}\left(y \odot b_{i}\right) \odot a_{i}}\left\{\bigwedge_{1}^{n}\left\{f\left(y_{i} \odot b_{i}\right) \wedge f\left(a_{i}\right)\right\}\right\} \\
& =\bigvee_{x \odot y \in \sum_{i=1}^{n} p_{i} \odot a_{i}}\left\{\bigwedge_{1}^{n}\left\{f\left(p_{i}\right) \wedge f\left(a_{i}\right)\right\}\right\}=(f \circ f)(x \odot y) \\
& =\bigwedge_{z \in x \odot y}(f \circ f)(z) .
\end{aligned}
$$

It means that $f \circ f$ is a fuzzy left hyperideal so that $f \circ f$ is a fuzzy hyperideal.

Theorem 3.18. Let $(R, \oplus, \odot)$ be an LA-semihyperring with left identity. If $f$ is a fuzzy left hyperideal of $R$ then $f \cup(f \circ \boldsymbol{R})$ is a fuzzy hyperideal.

Proof. We have $f$ is a fuzzy left hyperideal of $R$. Since $R$ is an LA-semihyperring then

$$
\begin{aligned}
(f \cup(f \circ \boldsymbol{R})) \circ \boldsymbol{R} & =(f \circ \boldsymbol{R}) \cup((f \circ \boldsymbol{R}) \circ \boldsymbol{R}) \\
& =(f \circ \boldsymbol{R}) \cup(\boldsymbol{R} \circ \boldsymbol{R}) \circ f) \\
& =(f \circ \boldsymbol{R}) \cup(\boldsymbol{R} \circ f) \subseteq(f \circ \boldsymbol{R}) \cup f \\
& \subseteq f \cup(f \circ \boldsymbol{R}) .
\end{aligned}
$$

With analog way to prove that $f \cup(f \circ \boldsymbol{R})$ is a fuzzy left hyperideal. 
Definition 3.19. Let $(R, \oplus, \odot)$ be an LA-semihyperring and $f \in F(R)$. For all $t \in[0,1]$, we have level set of $R$ as follows.

$$
f_{t}=\{x \mid x \in R, f(x) \geq t\}
$$

Definition 3.20. Let $(R, \oplus, \odot)$ be an LA-semihyperring and $A$ be a non empty subset of $R$. The characteristic function of $A$ is defined as follows.

$$
\begin{aligned}
\chi_{A} & : R \mapsto[0,1] \\
: & : \chi_{A}(x)= \begin{cases}1, & x \in A \\
0, & x \notin A\end{cases}
\end{aligned}
$$

Lemma 3.21. Let $(R, \oplus, \odot)$ be an LA-semihyperring and $A, B \in P^{*}(R)$ then the properties are satisfied.

- If $A \subseteq B$ then $\chi_{A} \subseteq \chi_{B}$

- $\chi_{A}+\chi_{B}=\chi_{A+B}$

- $\quad \chi_{A} \circ \chi_{B}=\chi_{A \circ B}$

- $\quad \chi_{A} \cup \chi_{B}=\chi_{A \cup B}$

- $\quad \chi_{A} \cap \chi_{B}=\chi_{A \cap B}$

Theorem 3.22. Let $(R, \oplus, \odot)$ be an LA-semihyperring and $f$ be a subset fuzzy of $R$. Then Subset fuzzy $f$ is a fuzzy LA-subsemihyperring (resp., left, right hyperideal) of $R$ if only if for every $t \in[0,1]$, level set $f_{t}$ is a fuzzy LA-subsemihyperring (resp., left,right hyperideal) of $R$

Proof. Let $f$ be a fuzzy left hyperideal of $R$ and $f_{t} \neq \varnothing$. Let $x, y \in f_{t}$ and $r \in R$. By Definition 3.19 we have $f(y) \geq t$ for any $t \in[0,1]$ such that and

$$
\bigwedge_{z \in x \oplus y} f(z)=f(x \oplus y) \geq \min \{f(x), f(y)\} \geq t
$$

$$
\bigwedge_{z \in r \odot y} f(z)=f(r \odot y) \geq f(y) \geq t .
$$

By Definition 3.19 we have $x \oplus y \in f_{t}$ and $x \odot y \in f_{t}$. Thus $f_{t}$ is a fuzzy left hyperideal of $R$.

Conversely, suppose $f_{t}$ is a fuzzy left hyperideal of $R$ for every $t \in[0,1]$. Let $x, y \in R$. Assume

$$
\bigwedge_{z \in x \oplus y} f(z)=f(x \oplus y)<\min \{f(x), f(y)\}
$$

choose $t \in[0,1]$ such that

$$
\bigwedge_{z \in x \oplus y} f(z)<t \leq \min \{f(x), f(y)\} .
$$

Then $x, y \in f_{t}$ so it must be $x \oplus y \in f_{t}$ but $\bigwedge_{z \in x \oplus y} f(z)<t \quad$ so that $x \oplus y \notin f_{t}$. That is contradiction so that the assumption is wrong. Thus, $\Lambda_{z \in x \oplus y} f(z) \geq \min \{f(x), f(y)\}$. Furthermore, we will prove that $\Lambda_{z \in x \odot y} f(z) \geq f(y)$. Assume

$$
\bigwedge_{z \in x \odot y} f(z)=f(x \odot y)<f(y) .
$$

For $t \in[0,1]$, we have

$$
\bigwedge_{z \in x \odot y} f(z)<t \leq f(y) .
$$

So that $y \in f_{t}$. Since $f_{t}$ is fuzzy left hyperideal of $R$ then for any $x \in R, \quad x * y \in \boldsymbol{R} \odot f_{t} \subseteq f_{t} \quad$ but $\Lambda_{z \in x \odot y} f(z)<t$ so that $x \odot y \notin f_{t}$. It is contradiction, so that the assumption is wrong. Hence, $\Lambda_{z \in x \odot y} f(z) \geq$ $f(y)$. So, $f$ is a fuzzy left hyperideal of $R$.
Theorem 3.23. Let $R \oplus, \odot$ ) be an LA-semihyperring. The following statements are true.

(i) A non empty subset $S$ is an LA-subsemihyperring of $R$ if only if $\chi_{S}$ is a fuzzy LA-subsemihyperring of $R$.

(ii) A non empty subset $I$ is a left hyperideal (resp., right hyperideal) of $R$ if only if $\chi_{I}$ is an fuzzy left hyperideal (resp., right hyperideal) of $R$.

Proof. i) $(\Rightarrow$ ) Let $S$ be an LA-subsemihyperring of $R$. Let $x, y \in R$. If $x \oplus y \notin S$ and $x \odot y \notin S$ then $\chi_{S}(x)=$ $0, \chi_{S}(y)=0, \chi_{S}(x \oplus y)=0$ and $\chi_{S}(x \odot y)=0$ such that

$$
\begin{aligned}
& \bigwedge_{z \in x \oplus y} \chi_{S}(z)=\chi_{S}(x \oplus y) \geq 0=\min \left\{\chi_{S}(x), \chi_{S}(y)\right\} \\
& \text { and } \\
& \bigwedge_{z \in x \odot y} \chi_{S}(z)=\chi_{S}(x \odot y) \geq 0=\min \left\{\chi_{S}(x), \chi_{S}(y)\right\} .
\end{aligned}
$$

If $x \oplus y \in S$ and $x \odot y \in S$ then $\chi_{S}(x)=1$ and $\chi_{S}(y)=1$ such that

$$
\bigwedge_{z \in x \oplus y} \chi_{S}(z)=1 \geq \min \left\{\chi_{S}(x), \chi_{S}(y)\right\}
$$

and

$$
\bigwedge_{z \in x \odot y} \chi_{S}(z)=1 \geq \min \left\{\chi_{S}(x), \chi_{S}(y)\right\} .
$$

Therefore, $\chi_{S}$ is a fuzzy LA-subsemihyperring of $R$. $(\Leftarrow)$ Let $\chi_{S}$ be a fuzzy LA-subsemihyperring of $R$. Let $x, y \in R$ such that $\chi_{S}(x)=1$ and $\chi_{S}(y)=1$ then

$$
\begin{aligned}
& \bigwedge_{z \in x \oplus y} \chi_{S}(z)=\chi_{S}(x \oplus y) \geq \min \left\{\chi_{S}(x), \chi_{S}(y)\right\}=1 \\
& \text { and } \\
& \bigwedge_{z \in x \odot y} \chi_{S}(z)=\chi_{S}(x \odot y) \geq \min \left\{\chi_{S}(x), \chi_{S}(y)\right\}=1
\end{aligned}
$$

Hence, $\chi_{S}(x \oplus y)=1$ and $\chi_{S}(x \odot y)=1$ such that $x \oplus y \in S$ and $x \odot y \in S$. Thus, $S$ is an LAsubsemihyperring of $R$.

ii) $(\Longrightarrow)$ Let $I$ be a left hyperideal of $R$. Let $x, y \in R$. If $y \notin I$ then $\chi_{I}(y)=0$ such that

$$
\bigwedge_{z \in x \odot y} \chi_{I}(z)=\chi_{I}(x \odot y) \geq 0=\chi_{I}(y) .
$$

If $y \in I$ then $x * y \in I$. Since $I$ is a left hyperideal of $R$ then

$$
\bigwedge_{z \in x \odot y} \chi_{I}(z)=\chi_{I}(x \odot y) \geq 1=\chi_{\mathrm{I}}(y) .
$$

So, $\chi_{I}$ is a fuzzy LA-subsemihyperring of $R$.

$(\Leftarrow)$ Let $\chi_{I}$ is a fuzzy LA-subsemihyperring of $R$. Let $r \in R$ and $y \in I$. Since $\chi_{I}$ is a fuzzy LAsubsemihyperring of $R$ then

$$
\bigwedge_{z \in r \odot y} \chi_{I}(z)=\chi_{I}(r \odot y) \geq \chi_{\mathrm{I}}(y)=1 .
$$

This implies $\chi_{I}(r \odot y)=1$ such that $r \odot y \in R \odot$ $I \subseteq I$. Hence, $I$ is a hyperideal of $R$.

\section{CONCLUSION}

The set of all fuzzy subsets of LA-semihyperring is also LA-semihyperring so that the algebraic hyperstructure satisfies all the axioms on the left almost 
structure. A fuzzy subset of an LA-semihyperring can be considered as fuzzy sub LA-semihyperring, fuzzy left hyperideal, or fuzzy right hyperideal so that all sub fuzzy and hyperideal fuzzy properties are fulfilled. A fuzzy subset is fuzzy sub LA-semihyperring (fuzzy hyperideal) if and only if the level set is also fuzzy sub LAsemihyperring (fuzzy hyperideal). A subset of LAsemihyperring (hyperideal) is sub LA-semihyperring (hyperideal) if and only if the characteristic function is also sub LA-semihyperring (hyperideal).

\section{ACKNOWLEDGMENTS}

The authors gratefully acknowledge the reviewer who has been willing to review our article. Your comments and suggestions have really helped in the development of our article.

\section{REFERENCES}

[1] F. Marty, "Sur une generalization de la notion de groupe," pp. 45-49.

[2] L. A. Zadeh, "Fuzzy sets," vol. 8, no. 3, pp. 338-353.

[3] A. Rosenfeld, "Fuzzy groups," vol. 35, no. 3, pp. 512-517.

[4] N. Kuroki, "On fuzzy semigroups," vol. 53, no. 3, pp. 203-236.

[5] P. Corsini and V. Leoreanu, Applications of Hyperstructure Theory. Dordrecht: Kluwer Academic, 2003.

[6] J. Ahsan, J. N. Mordeson, and M. Shabir, Fuzzy semirings with applications to automata theory, vol. 278. Springer.

[7] M. K. Sen, R. Ameri, and G. Chowdhury, "Fuzzy hypersemigroups," vol. 12, no. 9, pp. 891-900.

[8] T. K. Dutta, S. Kar, and S. Purkait, "Intervalvalued fuzzy prime and semiprime ideals of hypersemiring," Ann. Fuzzy Math. Informatics, vol. 9, no. January, pp. 261-278, 2015.

[9] N. Kehayopulu, "Hypersemigroups and fuzzy hypersemigroups," vol. 10, no. 5, pp. 929-945.

[10] S. Nawaz, I. Rehman, and M. Gulistan, "On left almost semihyperrings," vol. 16, no. 4, pp. 528541. 